\title{
Differential Expression of Correlates of Classical Conditioning in Identified Medial and Lateral Type A Photoreceptors of Hermissenda
}

\author{
Robert J. Frysztak and Terry Crow \\ Department of Neurobiology and Anatomy, University of Texas Medical School, Houston, Texas 77225
}

Classical conditioning of Hermissenda produces neurophysiological correlates in the primary sensory neurons of the pathway mediating the conditioned stimulus (CS), the type B and type A photoreceptors. Biophysical and biochemical changes intrinsic to the type $B$ photoreceptors have been studied extensively in conditioned animals. A second site for intrinsic modification with learning has been recently identified in type A photoreceptors. We have recorded from identified medial and lateral type A photoreceptors in conditioned animals and animals that received pseudorandom presentations of light (CS) and rotation (unconditioned stimulus). Here we report that conditioning produces differential effects upon CS-elicited spike frequency and intrinsic excitability detected in identified lateral and medial type $A$ photoreceptors. Lateral type A photoreceptors from conditioned animals exhibited significant increases in spike frequency elicited by the CS as compared to pseudorandom controls. In contrast, CS-elicited spike frequency recorded in medial type A photoreceptors was not significantly different from random controls. The amplitude of the peak and plateau phases of the generator potential of medial and lateral type A photoreceptors was decreased in conditioned animals as compared to random controls. In addition, only lateral type A photoreceptors exhibited enhanced cellular excitability as expressed by increased spike discharges produced by the injection of extrinsic depolarizing current pulses. Conditioning also decreased spike frequency accommodation in lateral type A photoreceptors. In normal controls, medial type B photoreceptors produced stronger synaptic inhibition of medial type $A$ photoreceptors than the lateral type B photoreceptors inhibition of lateral type A photoreceptors. The difference in the effectiveness of synaptic inhibition between identified pairs of type $B$ and type $A$ photoreceptors may contribute to the differential expression of correlates of conditioning found in medial and lateral type A photoreceptors.

[Key words: Hermissenda, classical conditioning, photoreceptor, enhanced excitability, associative learning, synaptic inhibition]

Received Nov. 6, 1992; revised Jan. 20, 1993; accepted Jan. 26, 1993.

This work was supported by NIMH Grant MH40860 (to T.C.) and NIMH Research Service Award MH10326 (to R.J.F.).

Correspondence should be addressed to Dr. Robert J. Frysztak, Department of Neurobiology and Anatomy, University of Texas Medical School, P.O. Box 20708, Houston, TX 77225 .

Copyright (C) 1993 Society for Neuroscience $0270-6474 / 93 / 132889-09 \$ 05.00 / 0$
Classical conditioning and its concomitant cellular neurophysiological correlates have been studied extensively in the nudibranch mollusk Hermissenda crassicornis (Crow and Alkon, 1978, 1980; Alkon et al., 1982, 1985; West et al., 1982; Crow, 1983, 1988; Farley and Alkon, 1985; Farley et al., 1990). Conditioning, consisting of paired presentations of light (conditioned stimulus, CS) and high-speed rotation (unconditioned stimulus, US) results in suppression of normally positive phototactic behavior (Crow and Alkon, 1978; Crow and Offenbach, 1983). Learning is expressed by changes in a number of locomotor responses, including an increase in the latency to initiate locomotion in the presence of the CS (Crow and Offenbach, 1983), increases in the latency to enter an illuminated area (Crow and Alkon, 1978), and contraction of the foot musculature elicited by the CS (Lederhendler et al., 1986).

Intrinsic cellular neurophysiological correlates have been identified in type B photoreceptors following conditioning using multiple trials of paired light and rotation (Crow and Alkon, 1980), and in animals that received one-trial conditioning (Crow and Forrester, 1991). These results have suggested that cellular correlates are expressed differentially in identified medial and lateral type B photoreceptors (Alkon et al., 1985; Crow and Forrester, 1991). In addition to the extensively studied correlates in identified type B photoreceptors, the type A photoreceptor has also been implicated as a locus for cellular changes produced by conditioning (Farley et al., 1990). CS-elicited reductions in spike frequency, a decrease in the amplitude of the plateau phase of generator potentials, and a reduction in darkadapted input resistance have been observed in type $\Lambda$ photoreceptors of conditioned animals (Farley and Alkon, 1982; Farley et al., 1990). These results suggest that cellular correlates of conditioning may be different in type A and type B photoreceptors, and may be expressed in both medial and lateral type A photoreceptors (Farley et al., 1990). However, since previous studies have focused upon an analysis of synaptically isolated photoreceptors, it has not been established if differences in excitability produced by conditioning are expressed in both medial and lateral type A photoreceptors. Studies of synaptically intact preparations are important, since previous research has suggested that inhibition of the medial type A photoreceptor by the medial type $\mathrm{B}$ photoreceptor following conditioning is a critical synaptic event in producing the reduced phototaxis of conditioned animals (Goh and Alkon, 1984; Goh et al., 1985). Here we report that correlates of conditioning are differentially expressed in identified type A photoreceptors. We found in synaptically intact preparations that CS-elicited spike activity is significantly increased only in the lateral type A photoreceptor. In addition, enhanced excitability and reduced accommodation 
were only observed in lateral type A photoreceptors. These results suggest that similar mechanisms may support conditioning correlates observed in both type B and type A photoreceptors.

A preliminary report of these results has been presented in abstract form (Frysztak and Crow, 1991).

\section{Materials and Methods}

Animals. Adult Hermissenda crassicornis were used in all experiments. The animals were obtained from Sea Life Supply, Sand City, CA, and maintained in closed artificial seawater aquaria at $14 \pm 1^{\circ} \mathrm{C}$ on a $12 \mathrm{hr}$ light/ 12 hr dark cycle. Amimals were fed small pieces of scallop daily. Behavioral training, testing, and electrophysiological procedures were performed during the light phase of the light/dark cycle.

Baseline test of phototactic behavior. The details of the conditioning procedures and methods for testing phototactic behavior have been described in detail in previous publications (Crow and Offenbach, 1983; Crow, 1985) and will be described only briefly in this report. Animals were tested prior to training to determine baseline latencies to initiate locomotion in response to a test light. Previous research has shown that the increase in the time taken by the animals to locomote into the test light can be accounted for by an increase in the latency to initiate locomotion (Crow and Offenbach, 1983). Animals were placed into glass tubes $228 \mathrm{~mm}$ long filled with artificial seawater. A foam plug inserted through an opening confined the animal to one end of the tube. The tubes were attached by spring clips to a modified turntable enclosed in an incubator maintained at $15^{\circ} \mathrm{C}$. A light spot $\left(10^{-4} \mathrm{~W} / \mathrm{cm}^{2}\right.$, white light was projected onto the center of the turntable, illuminating a circular area $15-16 \mathrm{~cm}$ in diameter. Animals were dark adapted for $12 \mathrm{~min}$ prior to testing phototactic behavior. The latencies to locomote in the presence of the test light were recorded when a Hermissenda moved between an infrared emitter and a phototransistor at the starting end of each glass tube. When the infrared beam was interrupted, a free-running digital clock was turned off and the time recorded for later data analysis. Animals that did not respond within a $20 \mathrm{~min}$ criterion period during the pretraining measurements were not used in the conditioning experiment.

Conditioning procedure. Following baseline measurements, animals were randomly assigned to paired and pseudorandom control groups. The conditioning phase consisted of 100 trials of the CS and US each day for 3 consecutive days. For the conditioned group, each trial consisted of a $10 \mathrm{sec} C S$ (light) paired with high-speed $(95 \mathrm{rpm})$ rotation (US), with an average intertrial interval of $90 \mathrm{sec}$. The intensity of the CS was the same as the test light used to establish baseline responding of phototactic behavior during the pretest condition. The pseudorandom control group received 100 trials each day for 3 consecutive days, consisting of light and rotation programmed on explicitly unpaired, pseudorandom schedules. Animals were returned to their home cages between training sessions.

Postacquisition test. All animals underwent behavioral testing identical to the pretraining (baseline) test measurement for phototaxis $24 \mathrm{hr}$ after the third conditioning session. Animals that did not initiate locomotion in the presence of the CS within 20 min during the post-test received a maximum latency score. Retention of conditioning was in the form of suppression ratios comparing posttraining behavior with pretraining test scores. The ratio is expressed as $A / A+B$, where $A$ represents pretraining scores and $B$ represents posttraining scores. Ratios less than 0.5 indicate an increase in start latencies in the presence of the CS following conditioning.

Intracellular recordings from identified type A photoreceptors. Intracellular recordings from identified type A photoreceptors were collected for both groups (paired and pseudorandom control) 24 or $48 \mathrm{hr}$ after the last training session. Anatomical and electrophysiological criteria were used to identify specific type A and B photoreceptors within the eye. Identified type A photoreceptors are located in the anterior-medial and anterior-lateral portions of the eye near the lens, and type B photoreceptors are located in the posterior-lateral and posterior-medial portions of the eye as previously reported (Alkon and Fuortes, 1972). In addition, type $\mathrm{A}$ photoreceptors typically are not spontaneously active in the dark, and have larger-amplitude action potentials in comparison to type B photoreceptors. However, action potential amplitudes $>20$ $\mathrm{mV}$ clearly do not distinguish type A from type $\mathrm{B}$ photoreceptors, as previously suggested (Farley et al., 1990). Simultaneous recordings from pairs of type $A$ and type $B$ photoreceptors where there is no ambiguity as to the type of photoreceptor impaled revealed an average type B spike amplitude of $20.05 \mathrm{mV} \pm 4.03(\mathrm{SD}) ; N=60$; range $=14-30 \mathrm{mV}$.

The circumesophageal nervous system was removed from the animal and pinned to an elevated stage in a recording chamber lined with Sylgard (Dow Chemical). The chamber was filled with artificial seawater of the following composition (in mM): $460 \mathrm{NaCl}, 10 \mathrm{KCl}, 10 \mathrm{CaCl}_{2}, 55$ $\mathrm{MgCl}_{2}$; buffered with $10 \mathrm{mM}$ HEPES and brought to $\mathrm{pH} 7.46$ with dilute $\mathrm{NaOH}$. The isolated nervous system was incubated in a protease solution (Sigma; $0.67 \mathrm{mg} / \mathrm{ml}, 5-7 \mathrm{~min}$ ) to facilitate microelectrode penetration. Identified type A photoreceptors were impaled with glass microelectrodes filled with $4.0 \mathrm{M}$ potassium acetate, with resistances of 30-50 M . The electrodes were connected via a silver wire to the head stage of a Getting microelectrode amplifier. Intracellular recordings were monitored on a Tektronix storage oscilloscope, and recorded on a Gould 3400 brush pen recorder. The artificial seawater in the recording chamber was monitored by a thermistor and held constant at $15 \pm 0.5^{\circ} \mathrm{C}$.

Illumination of the isolated nervous system was provided by a tungsten halogen incandescent lamp attached to a fiber-optic bundle mounted underncath the recording chamber. The nervous system was illuminated by white light. The light was attenuated as desired by inserting neutral density filters into the path of the beam. Light intensities are described in log units of attenuation, neutral densities (ND). Neurophysiological experiments were carried out using light stimuli of the same intensities that were used in behavioral experiments.

Current-elicited activity. Photoreceptors were dark adapted for $12 \mathrm{~min}$ before collection of electrophysiological data. Spike frequency accommodation to extrinsic depolarizing current injection was determined during the last $5 \mathrm{~min}$ of dark adaptation. Depolarizing pulses of 20-40 $\mathrm{mV}$ (in $5 \mathrm{mV}$ steps) were injected through the recording electrode for $20 \mathrm{sec}$ with an interval between successive current injections of at least $2 \mathrm{~min}$. Spike frequency was determined for each $5 \mathrm{sec}$ bin during the injection period. Excitability measurements to extrinsic current were determined from the initial 5 sec of current injection. Accommodation to the extrinsic current pulse was determined by comparing the spike frequency of the first $5 \mathrm{sec}$ (seconds 1-5) of current injection to the subsequent 5 sec (seconds 6-10).

CS-elicited activity. Following dark adaptation and current injection, the response to light of identified type A photoreceptors was examined during the first $30 \mathrm{sec}$ of a 5 min light step at five different levels of light intensity: $-4,-3,-2,-1$, and $0 \mathrm{ND}$. The five intensities were presented in an ascending series for each of the identified (medial or lateral) type A photoreceptors used in the analysis. Following measurements at the initial light intensity $(-4 \mathrm{ND})$, the photoreceptors were dark adapted for an additional $6 \mathrm{~min}$ before presenting the next higher intensity. The generator potential amplitude at $30 \mathrm{sec}$ after light onset was determined from the Gould recordings by measuring the amplitude in light from the dark-adapted resting potential. Spike frequency was also determined from these recordings by counting the number of action potentials during the plateau phase (last $25 \mathrm{sec}$ of a $30 \mathrm{sec}$ light step) of the generator potential, and during the last $30 \mathrm{sec}$ of a $5 \mathrm{~min}$ light step. The peak generator potential amplitude represents the largest amplitude of the generator potential elicited during the first $30 \mathrm{sec}$ of the light step. Generator potential amplitudes during the plateau phase of the light response were also determined at $60 \mathrm{sec}, 120 \mathrm{sec}$, and during the last 30 sec of a 5 min light step for each of the five light intensities studied by measuring the peak amplitude in light from the dark-adapted resting potential at each of these points.

Statistical analysis. A Mann-Whitney $U$ test for large samples was used to assess the effects of conditioning on the phototactic behavior of the animals since cutoff scores were used during the postconditioning measurement of photutactic latencies. Since no significant differences in electrophysiological measures were found at 24 compared to $48 \mathrm{hr}$ after the last training session, the data from the two groups were combined for the statistical analysis.

A treatment by trials analysis of variance was used to examine overall differences between conditioned and pseudorandom groups at the different light intensities (Winer, 1962). Following significant effects of treatment, individual comparisons between selected groups were performed using two-tailed $t$ tests.

\section{Results}

\section{Conditioning}

Pairings of light (CS) with rotation (US) produced phototactic suppression that was similar in magnitude to previously pub- 

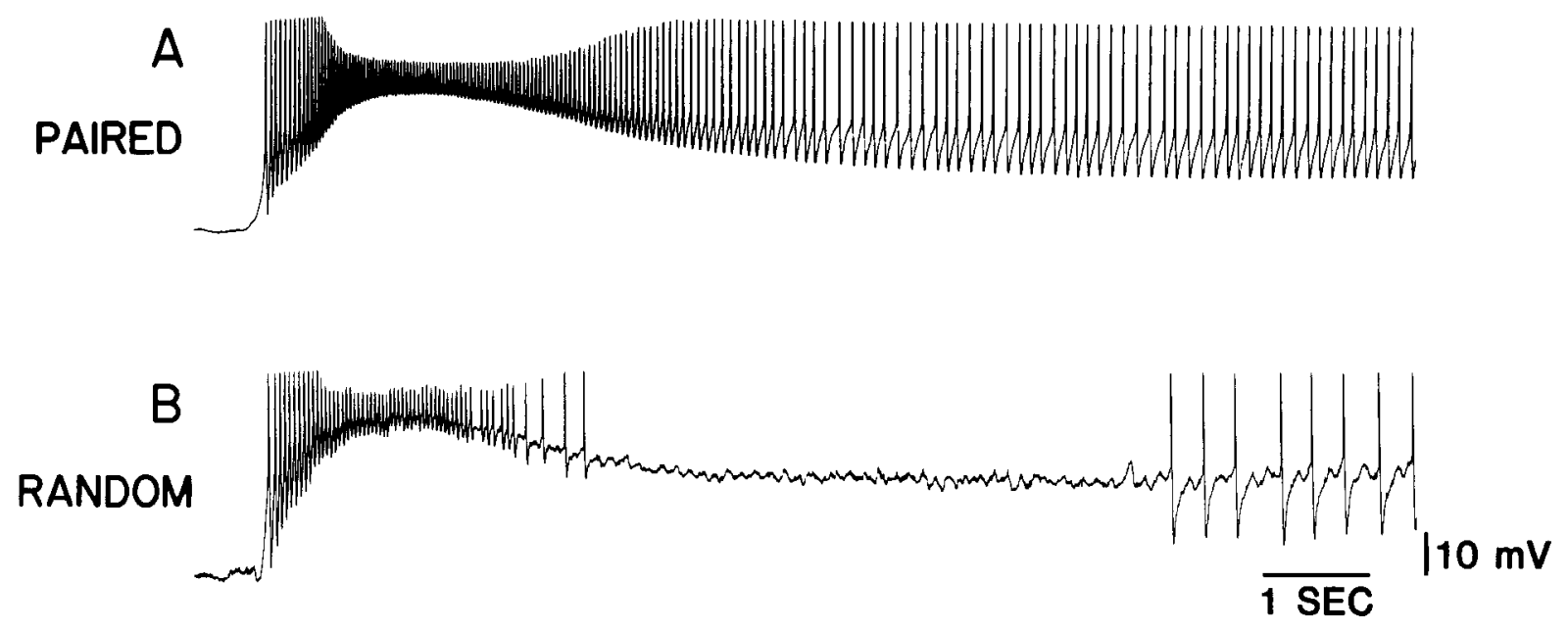

Figure 1. Representative examples of generator potentials recorded from lateral type A photoreceptors during the first 10 sec of a 5 min light step (light attenuated $2 \log$ units) in Hermissenda that received either paired $(A)$ or pseudorandom $(B)$ presentations of light (CS) and rotation (US). Conditioning resulted in an increase in spike frequency and a reduction in the transient peak of the generator potential $(A)$ as compared to the example recorded from a preparation that received the pseudorandom control procedure $(B)$.

lished reports (Crow and Alkon, 1978; Crow and Offenbach, 1983). The electrophysiological analysis was conducted with animals 24 and 48 hr after conditioning. Conditioning produced significantly greater suppression for paired animals as compared to controls $(z=3.89 ; p<0.001)$.

\section{Cellular correlates in identified type A photoreceptors}

CS-elicited spike frequency. Intracellular recordings were obtained from identified medial and lateral type A photoreceptors in isolated circumesophageal nervous systems from conditioned and pseudorandom control animals. Type A photoreceptors are located in the anterior portion of the eye, and were anatomically and electrophysiologically identified as either medial or lateral as previously described. Analysis of the electrophysiological data revealed statistically significant differences in CS-elicited spike frequency during the plateau phase of the generator potential between conditioned and pseudorandom control animals. Figure 1 illustrates representative examples of light-elicited $(-2$ ND) generator potentials recorded from lateral type A photoreceptors in a conditioned $(A)$ and a pseudorandom control animal $(B)$. As shown in Figure 1, conditioning resulted in an increase in light-elicited spike frequency of a lateral type $A$ photoreceptor during the plateau phase of the generator potential as compared to the example from the pseudorandom control. The increase in light-elicited spike frequency was observed in cases where the generator potential amplitude was smaller in the paired group as compared to the pseudorandom control.

Group data depicting the mean spike frequency elicited by four different light steps during the plateau phase (between 5 and $30 \mathrm{sec}$ after light onset) of the generator potential in identified medial and lateral type A photoreceptors from conditioned and pseudorandom controls are shown in Figure 2. Conditioning resulted in differential effects upon CS-elicited spike frequency in identified medial and lateral type A photoreceptors. The results of the ANOVA showed significant overall effects of treatment only for the lateral type $A$ photoreceptors $\left(F_{1,21}=19.49\right.$; $p<0.01$ ). Individual paired comparisons between conditioned and pseudorandom controls showed that the CS elicited significantly more action potentials in conditioned animals than in pseudorandom controls at moderate and bright light intensities $\left(-2 \mathrm{ND}, t_{21}=2.64, p<0.01 ;-1 \mathrm{ND}, t_{19}=2.27, p<0.05 ; 0\right.$ $\left.\mathrm{ND}, t_{16}=2.29, p<0.05\right)$ in the lateral type A photoreceptor. In contrast to the effects of conditioning on activity in the lateral type A photoreceptor, mean CS-elicited spike frequencies recorded from medial type A photorcceptors from the conditioned group were not significantly different from pseudorandom controls at any of the four light intensities studied. These results show that conditioning produced effects on CS-elicited spike frequency that were only expressed in the lateral type A photoreceptor. Previously, it was reported that under steady-state light-adapted conditions, type $B$ photoreceptors of conditioned animals exhibited a decrease in CS-elicited spike frequency
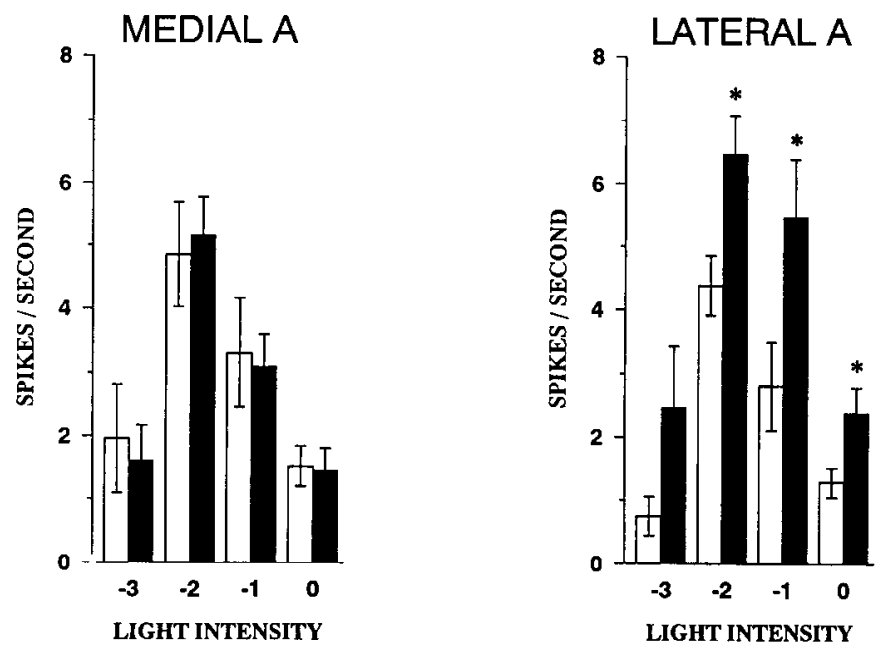

Figure 2. Group data showing the mean spike frequency elicited by a $30 \mathrm{sec}$ light step in identified medial and lateral type A photoreceptors. Data were collected during the plateau phase of the generator potential (seconds 5-30). Solid bars denote mean responses ( \pm SEM) recorded from conditioned animals; open bars denote mean responses ( \pm SEM) recorded from pseudorandom controls. Four different light intensities were examined for both groups. Lateral type A photoreceptors of conditioned animals exhibited significant increases in spike frequency as compared to pseudorandom controls $\left(^{*}, p<0.05\right)$. In contrast, spike frequencies recorded from medial type A photoreceptors of conditioned animals were not significantly different from pseudorandom controls at any of the light intensities studied. 
Table 1. Light-adapted mean spike frequency

\begin{tabular}{|c|c|c|c|c|c|c|c|c|}
\hline & \multicolumn{4}{|c|}{ Medial type A photoreceptors } & \multicolumn{4}{|c|}{ Lateral type A photoreceptors } \\
\hline & $\overline{-3}$ & -2 & -1 & 0 & -3 & -2 & -1 & 0 \\
\hline Paired & $0.25 \pm 0.10(15)$ & $0.86 \pm 0.16(15)$ & $0.70 \pm 0.19(13)$ & $0.26 \pm 0.11(9)$ & $0.23 \pm 0.11(12)$ & $1.07 \pm 0.17(12)$ & $1.21 \pm 0.26(11)$ & $0.45 \pm 0.14(10)$ \\
\hline Pseudorandom & $0.38 \pm 0.17$ & $1.06 \pm 0.27$ & $0.88 \pm 0.19$ & $0.34 \pm 0.21(5)$ & $0.23 \pm 0.12(11)$ & $0.92 \pm 0.16(11)$ & $1.09 \pm 0.24(10)$ & $0.39 \pm 0.12$ \\
\hline
\end{tabular}

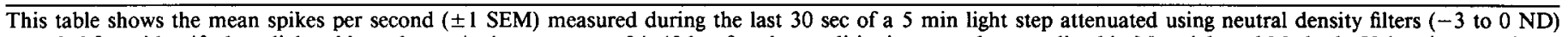

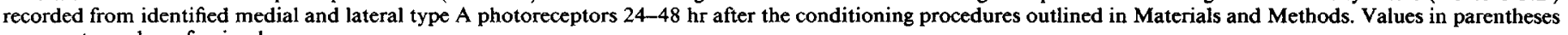
represent number of animals.

(Crow, 1983). In the present study, this was examined by determining CS-elicited spike frequency of identified type A photoreceptors under steady-state light-adapted conditions ( $\sim 5 \mathrm{~min})$. Group data showing the mean spike frequency elicited by four different light intensities during the last $30 \mathrm{sec}$ of a $5 \mathrm{~min}$ light step in identified medial and lateral type A photoreceptors are shown in Table 1. In contrast to the activity of B photoreceptors after conditioning, neither medial nor lateral type A photoreceptors exhibited significant differences in spike frequency under light-adapted conditions following pairing as compared to pseudorandom controls.

Amplitude of CS-elicited generator potentials: peak. Intracellular recordings were obtained from dark-adapted, identified medial and lateral type A photoreceptors from conditioned and pseudorandom control animals. Group data showing the mean peak amplitude of generator potentials elicited by five different light intensities are shown in Table 2. The results of two-group comparisons revealed that the generator potential peak in response to the brightest light was significantly smaller following conditioning for both medial $\left(t_{15}=2.36 ; p<0.05\right)$ and lateral $\left(t_{17}=2.82 ; p<0.05\right)$ type A photoreceptors as compared to pseudorandom controls. These results show that conditioning resulted in a decrease in the transient peak of the generator potential elicited by the brightest light intensity as compared to pseudorandom controls.

Amplitude of CS-elicited generator potentials: plateau. Group data showing the mean amplitude of generator potentials elicited by five different light intensities recorded from identified medial and lateral type A photoreceptors at the end of a $30 \mathrm{sec}$ light step from conditioned and pseudorandom control animals are shown in Figure 3. The ANOVA revealed significant overall effects of treatment for the medial type A photoreceptors $\left(F_{1,21}\right.$ $=9.94 ; p<0.01)$. The overall effects were not significant for the lateral A photoreceptor $\left(F_{1,21}=3.65\right.$, NS). However, two paired statistical comparisons were conducted, since they were planned in advance of the overall $F$ test and based upon previous work (Farley and Alkon, 1982) showing reductions in the generator potential amplitudes at these light intensities with conditioning. Individual paired comparisons between conditioned and pseudorandom controls showed that the amplitude of the generator potential in conditioned animals was significantly less than pseudorandom controls at virtually all light intensities $(-3$ $\mathrm{ND}, t_{21}=3.14, p<0.01 ;-2 \mathrm{ND}, t_{21}=2.70, p<0.025 ;-1$ $\mathrm{ND}, t_{19}=2.27, p<0.05 ; 0 \mathrm{ND}, t_{15}=2.50, p<0.025$ ) for medial type $A$ photoreceptors, and at moderate to bright intensities $\left(-1 \mathrm{ND}, t_{19}=2.62, p<0.025 ; 0 \mathrm{ND}, t_{16}=5.48, p<\right.$ 0.01 ) for lateral type A photoreceptors.

Group data showing the mean amplitude of the generator potential elicited by five different light intensities recorded at different times following light onset in medial and lateral type A photoreceptors from conditioned and pseudorandom controls are shown in Table 2. The mean amplitude of the generator potential was measured at four time points (peak, $60 \mathrm{sec}, 120$ $\mathrm{sec}$, and $5 \mathrm{~min}$ ) during a $5 \mathrm{~min}$ light step. Individual paired comparisons between conditioned and pseudorandom controls revealed that the amplitude of the generator potential was significantly smaller at the brightest light intensity $(0 \mathrm{ND})$ following conditioning at all four time points studied for the medial type

Table 2. Mean amplitude of the generator potential-peak and plateau phase

Medial type A photoreceptors

\begin{tabular}{lllllll}
\hline-4 & -3 & -2 & -1 & 0 & $\frac{\text { Lateral type A photoreceptors }}{-4}$ & -3
\end{tabular}

Peak

Paired $\quad 3.77 \pm 0.49(14) \quad 14.86 \pm 1.21(14) \quad 29.93 \pm 1.47(14) \quad 39.25 \pm 1.90(12) \quad 39.33^{*} \pm 2.13(9)$

$\begin{array}{lllllllll}\text { Pseudorandom } & 5.44 \pm 0.94 & \text { (9) } & 18.44 \pm 1.07 & \text { (9) } & 33.11 \pm 1.21 & \text { (9) } 42.33 \pm 0.96 & \text { (9) } 46.63 \pm 0.69(8)\end{array}$

$3.00 \pm 0.33(12) \quad 13.75 \pm 1.27(12)$

$60 \mathrm{sec}$

Paired $\quad 2.77 \pm 0.37(14) \quad 10.64 \pm 0.86(14) \quad 21.39 \pm 0.96(13) \quad 23.92 \pm 0.92(12) \quad 24.63^{*} \pm 1.25(8)$

$4.73 \pm 0.76(11) \quad 13.73 \pm 1.08(11)$

$\begin{array}{lllllllll}\text { Pseudorandom } & 3.78 \pm 0.72 & \text { (9) } & 14.33 \pm 1.29 & \text { (9) } 23.78 \pm 0.99 & \text { (9) } 25.43 \pm 0.47 & \text { (7) } 28.86 \pm 0.92 \text { (7) }\end{array}$

$1.92 \pm 0.30(12) \quad 11.30 \pm 0.78(12)$

$120 \mathrm{sec}$
Paired
$2.69 \pm 0.45(14) \quad 10.23 \pm 0.76(13)$
$18.64 \pm 1.03(12) \quad 22.33 \pm 0.76(12) \quad 20.88^{*} \pm 0.92(8)$
Pseudo
$\begin{array}{ll}2.69 \pm 0.45(14) & 10.2 \\ & 3.75 \pm 0.83\end{array}$
(9) $21.20 \pm 0.93$
(5) $23.86 \pm 0.86$
(7) $25.80 \pm 1.00(5)$
$1.83 \pm 0.31(12) \quad 11.11 \pm 0.76(10)$
$2.55 \pm 0.54(11) \quad 9.90 \pm 0.77(10)$

$2.64 \pm 0.52(11) \quad 10.18 \pm 0.81$ (11)

$5 \mathrm{~min}$

Paired

$3.15 \pm 0.45(14)$

Pseudorandom $3.00 \pm 0.91$

(14) $10.43 \pm 0.75(14) \quad 19.14 \pm 0.95$ (14) $22.50 \pm 0.89$ (12) $19.25^{*} \pm 0.72(8)$

$2.17 \pm 0.33(12) \quad 10.75 \pm 1.18(12)$

$2.46 \pm 0.56(11) \quad 10.27 \pm 0.71(11)$

This table shows the mean amplitude $(\mathrm{mV} \pm$ SEM) of the generator potential at the transient peak $(0-30 \mathrm{sec})$, and at $60 \mathrm{sec}, 120 \mathrm{sec}$, and $5 \mathrm{~min}$ after light onset $(-4$ to $0 \mathrm{ND}$ ) during the plateau phase evoked by a $5 \mathrm{~min}$ light step recorded from identified medial and lateral type A photoreceptors $24-48 \mathrm{hr}$ after the conditioning procedures descrihed in Materials and Methods. Values in parentheses represent number of animals.

*, Paired significantly less than pseudorandom $(p<0.05)$. 

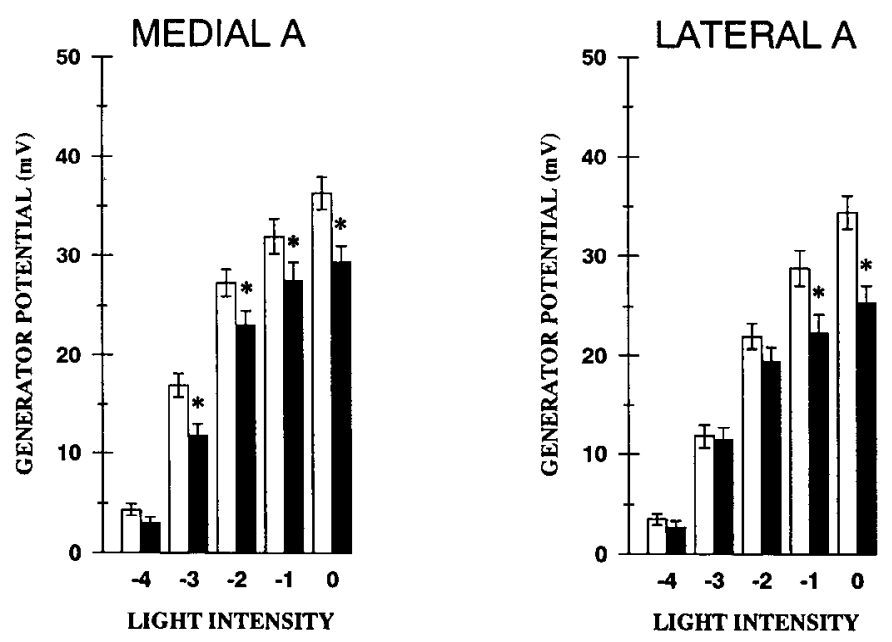

Figure 3. Group data showing mean amplitude of the plateau phase (30 sec after light onset) of generator potentials in identified medial and lateral type A photoreceptors. Solid bars denote mean responses ( \pm SEM) from conditioned animals; open bars denote mean responses ( \pm SEM) from pseudorandom controls. Conditioning resulted in significant decreases in the amplitude of the plateau phase of the generator potential in medial and lateral type $A$ photoreceptors as compared to pseudorandom controls $\left(^{*}, p<0.05\right)$.

A photoreceptor (peak, $t_{15}=2.36 ; 60 \mathrm{sec}, t_{12}=2.65 ; 120 \mathrm{sec}$, $\left.t_{11}=2.42 ; 5 \mathrm{~min}, t_{10}=2.98\right)$, and at the peak $\left(t_{17}=2.82\right)$ and $30 \mathrm{sec}\left(t_{15}=2.16\right)$ time points in the lateral type A photoreceptor as compared to pseudorandom controls $(p<0.05)$. These results show that conditioning resulted in a decrease in the amplitude of the generator potential as compared to pseudorandom controls. These data support previously reported studies describing reductions in the generator potential amplitude following conditioning (Farley et al., 1990).

Responses of type A photoreceptors to extrinsic current. Examples of intracellular recordings from lateral type A photoreceptors of conditioned and pseudorandom controls during a $20 \mathrm{sec}$ depolarizing current step are shown in Figure 4. Enhanced

Table 2. Extended

Lateral type A photoreceptors

$\begin{array}{lll}-2 & -1 & 0\end{array}$

Peak

Paired $\quad 24.33 \pm 1.18$ (12) $31.73^{*} \pm 1.10$ (11) $36.70^{*} \pm 1.55$ (10)

Pseudorandom $27.00 \pm 1.22$ (11) $36.30 \pm 1.39$ (10) $42.44 \pm 0.93$ (9)

$60 \mathrm{sec}$

Paired $\quad 18.00 \pm 1.01$ (12) $20.90 \pm 1.00$ (11) $21.89^{*} \pm 1.32$ (9)

Pseudorandom $19.18 \pm 0.83$ (11) $23.22 \pm 0.98$ (9) $26.25 \pm 0.90 \quad$ (8)

$120 \mathrm{sec}$

Paired $\quad 17.70 \pm 0.83(10) 19.80 \pm 0.96(10) 20.13 \pm 1.08$

Pseudorandom $18.46 \pm 0.78$ (11) $22.11 \pm 0.82$ (10) $22.57 \pm 1.25$ (8)

$5 \mathrm{~min}$

Paired $\quad 17.27 \pm 0.84$ (11) $20.70 \pm 0.89$ (10) $20.25 \pm 1.37 \quad$ (8)

Pseudorandom $18.82 \pm 0.85$ (11) $22.20 \pm 0.68$ (10) $21.75 \pm 1.13$ (8)
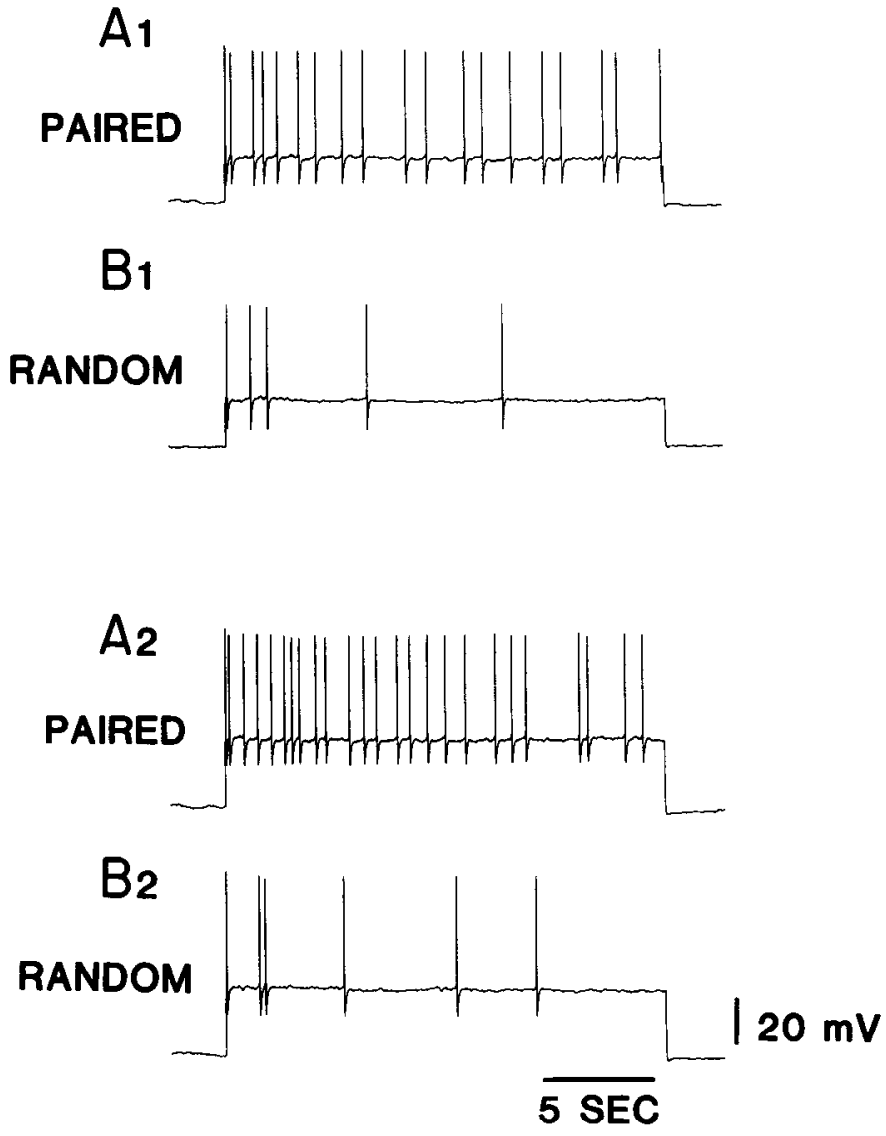

Figure 4. Representative examples of spike activity elicited by $20 \mathrm{sec}$ depolarizing extrinsic current steps in lateral type A photoreceptors from conditioned animals $(A I$ and $A 2)$ and pseudorandom controls $(B I$ and $B 2$ ). Recordings were collected from cells depolarized $20 \mathrm{mV}$ in $A l$ and $B 1$, and $30 \mathrm{mV}$ in $A 2$ and $B 2$. The spike frequency in the example from the conditioned group depolarized $20 \mathrm{mV}(A I)$ is enhanced as compared to the pseudorandom control $(B I)$. The spike frequency in the conditioned group depolarized $30 \mathrm{mV}(A 2)$ is again enhanced as compared to the pseudorandom control (B2). Increases in the level of depolarization in the examples from the conditioned group ( $A 1$ vs $A 2)$ resulted in an increase in spike frequency, whereas the same increase in the pseudorandom control group ( $B 1$ vs $B 2)$ resulted in virtually the same spike frequency.

excitability was expressed in conditioned lateral type A photoreceptors at all levels of depolarization examined.

Group data showing the mean spike frequency during the first $5 \mathrm{sec}$ of five different levels of current injection in medial and lateral type A photoreceptors are shown in Figure 5. Injection of depolarizing current into medial and lateral type A photoreceptors from animals in the pseudorandom control group resulted in similar spike frequencies $(0.25-1.00 \mathrm{spikes} / \mathrm{sec})$. ANOVA revealed significant overall effects of treatment (enhanced excitability) for lateral type A photoreceptors from the conditioned group as compared to the pseudorandom control group $\left(F_{1,29}=25.96 ; p<0.001\right)$. Individual paired comparisons between conditioned and pseudorandom controls were significant for all levels of depolarization studied $\left(20 \mathrm{mV}, t_{26}=2.94, p<\right.$ $0.01 ; 25 \mathrm{mV}, t_{20}=3.50, p<0.005 ; 30 \mathrm{mV}, t_{26}=4.52, p<$ $0.005 ; 35 \mathrm{mV}, t_{22}=5.90, p<0.005 ; 40 \mathrm{mV}, t_{23}=4.10, p<$ 0.005 ). In contrast, medial type A photoreceptors from the conditioned group were not significantly different from pseudorandom controls at any of the voltage levels studied, but did consistently exhibit a slight increase. 


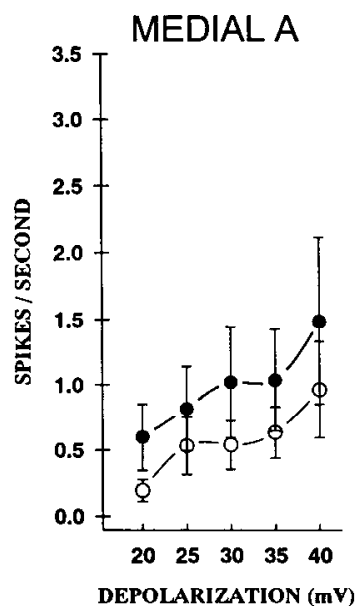

Figure 5. Group data showing mean spike frequency elicited by passage of extrinsic depolarizing current pulses in identified medial and lateral type A photoreceptors. Data were collected during the first $5 \mathrm{sec}$ of a 20 sec current step. Solid circles denote mean responses $( \pm$ SEM) recorded from conditioncd animals; open circles denote mean responses $( \pm$ SEM) recorded from pseudorandom controls. Spike frequencies recorded from medial and lateral type A photoreceptors of pseudorandom controls were similar for each of the five voltage levels examined. Spike frequencies from medial type A photoreceptors of conditioned animals were not significantly different from controls at any of the voltage levels studied. In contrast, spike frequencies from lateral type A photoreceptors of conditioned animals showed statistically significant increases in excitability as compared to pseudorandom controls at all voltage levels $(*, p<0.05)$.

Spike frequency accommodation was examined at five different levels of depolarization from the dark-adapted resting potential in conditioned and pseudorandom controls. Spike frequency accommodation to extrinsic current in the lateral $\mathrm{A}$ photoreceptor, expressed as the percentage decrease in spike frequency from the first $5 \mathrm{sec}$ to the subsequent $5 \mathrm{sec}$ of current injection, is shown in Figure 6. Lateral type A photoreceptors

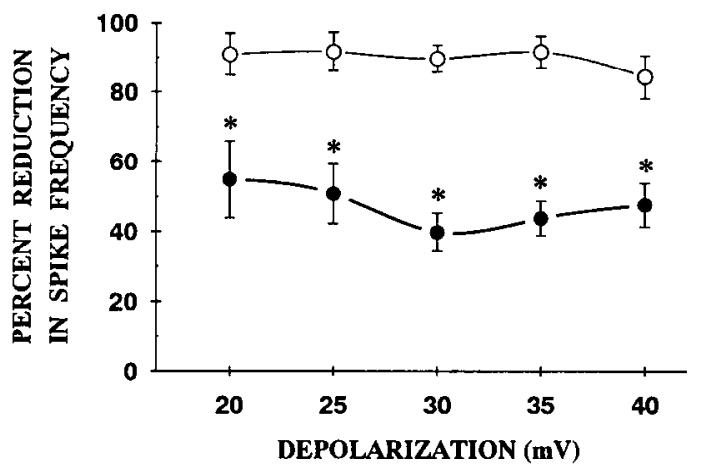

Figure 6. Group data showing spike frequency accommodation to extrinsic current in lateral type A photoreceptors. Values are expressed as the mean percentage decrease in spike frequency between the first 5 $\mathrm{sec}$ of extrinsic current injection and the subsequent $5 \mathrm{sec}$ during five different $20 \mathrm{sec}$ current pulses. Solid circles denote mean responses $( \pm$ SEM) recorded from conditioned animals; open circles denote mean responses $( \pm$ SEM) recorded from pseudorandom controls. Spike frequencies in lateral A photoreceptors from pseudorandom controls were reduced an average of $89.89 \pm 2.24 \%$. In contrast, spike frequencies in lateral A photoreceptors from conditioned animals were reduced an average of only $46.11 \pm 3.45 \%$. The reduction in accommodation of the conditioned group was significantly different from controls at all voltage levels examined $(*, p<0.05)$.
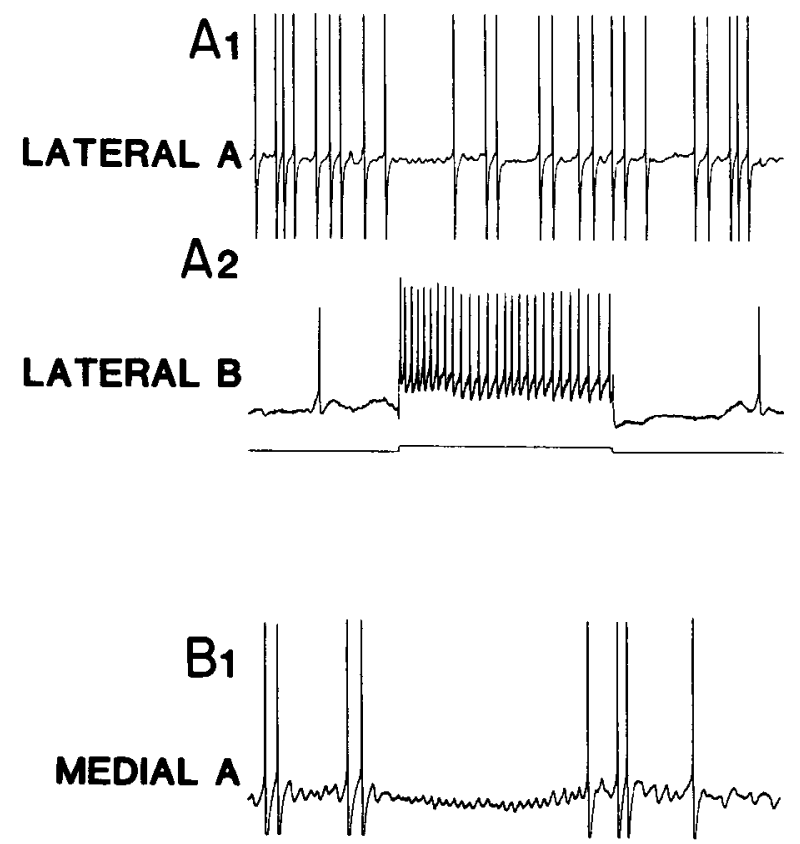

B2

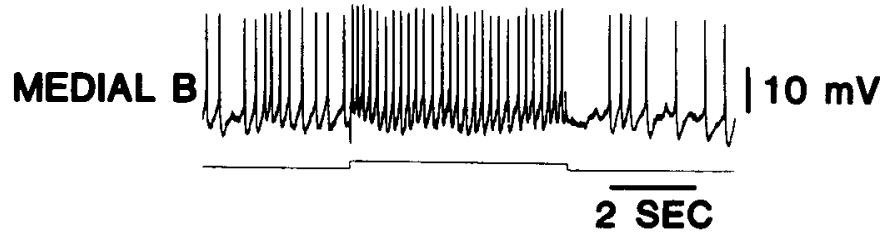

Figure 7. Representative examples of simultaneous recordings from lateral $(A I)$ and medial $(B I)$ type A photoreceptors and lateral $(A 2)$ and medial $(B 2)$ type B photoreceptors. Spikes were elicited in the normally quiescent type A photoreceptor by a $20 \mathrm{mV}$ depolarization from the resting potential. Type B photoreceptors were stimulated with a $5 \mathrm{scc}$ depolarizing current step. In the lateral A-B pair, increased firing of the lateral $\mathrm{B}$ photoreceptor $(A 2)$ had only a small effect on the spike discharge rate of the lateral A photoreceptor $(A I)$. In the medial A-B pair, however, increased firing of the medial $B$ photoreceptor $(B 2)$ produced strong inhibition of the medial A photoreceptor (BI). Also note the slight hyperpolarization of the membrane potential and the one-for-one occurrence of IPSPs in the medial type A photoreceptor and spikes in the type B photoreceptor. [Note: afterpotentials in the medial type A photoreceptor $(B I)$ appear smaller than those of the lateral type A photoreceptor $(A I)$ due to clipping by the Gould recorder.]

from the conditioned group showed significantly less accommodation of spike frequency at all voltage levels examined compared to pseudorandom controls. In contrast, medial type A photoreceptors from the conditioned group did not show significant changes in accommodation to any of the voltage levels examined.

\section{Synaptic interactions between pairs of identified $A$ and $B$ photoreceptors: synaptic strength}

Intracellular recordings were obtained from untrained (naive), dark-adapted pairs of lateral type $A$ and $B$ photoreceptors and medial type A and B photoreceptors. To study the strength of the synaptic connections, steady depolarizing current was injected into an identified type A photoreceptor $(10-20 \mathrm{mV})$ in order to produce action potentials in the normally quiescent $A$ cell. Depolarizing current was then passed for $5 \mathrm{sec}$ in the corresponding $B$ photoreceptor to increase the frequency of firing to approximately 6 spikes/sec. Figure 7 displays representative 
A

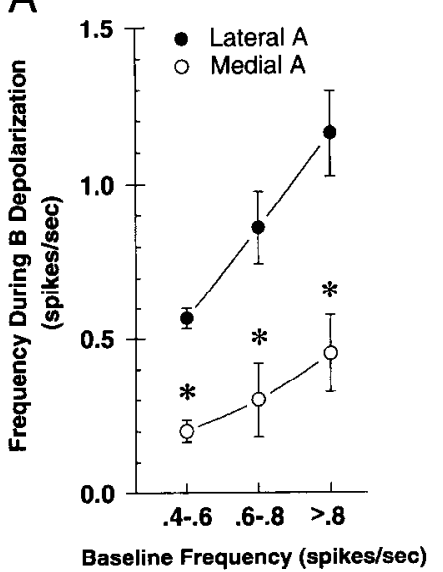

B

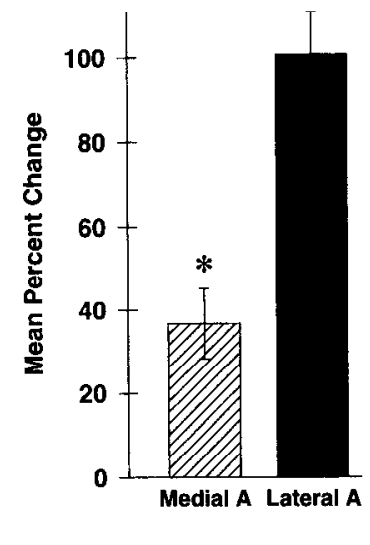

Figure 8. Strength of synaptic connections between identified pairs of type B and type A photoreceptors. $A$, Group data showing the mean spike frequency in the lateral and medial type A photoreceptors during the depolarizing current step $(5 \mathrm{sec})$ in the lateral or medial type B photoreceptor over various ranges of baseline spike frequencics in the A photoreceptors. Spike frequency values for the medial A photoreceptor were significantly smaller at all baseline frequencies tested as compared to the lateral A photoreceptor $\left({ }^{*}, p<0.01\right)$, indicating a strong inhibitory effect by the medial B photoreceptor. $B$, Group data showing the mean percentage change in the spike discharge rate of identified medial and lateral type A photoreceptors during stimulation of the corresponding medial or lateral type B photoreceptor. Depolarization of the medial $B$ photoreceptor resulted in a significant decrease $(63 \%)$ in spike frequency of the medial A photoreceptor $(n=6)$ as compared to the spike frequency of the lateral A photoreceptor $(n=5)$ during depolarization of the lateral B photoreceptor $\left({ }^{*}, p<0.001\right)$.

recordings from a lateral A-B pair and a medial A-B pair of photoreceptors. In the lateral pair, depolarization of the lateral $\mathrm{B}$ photoreceptor $(A 2)$ resulted in only modest or slight inhibition of spike frequency in the lateral A photoreceptor $(A l)$ at the onset of the current pulse. IPSP frequency in the A cell was also modest. In contrast, depolarization of the medial B photoreceptor $(B 2)$ resulted in a pronounced reduction in the number of action potentials elicited in the medial A photoreceptor $(B I)$. In addition, IPSP activity recorded in the medial A followed the medial B action potentials "one for one."

Group data showing the mean frequency of firing in the A photoreceptors during depolarizing stimulation of the type B photoreceptor are shown in Figure $8 A$. Steady extrinsic depolarizing current elicited action potentials in identified type A photoreceptors in a range of frequencies. The ANOVA revealed significant overall differences between lateral and medial type A photoreceptors $\left(F_{1,46}=28.34, p<0.001\right)$. Individual paired comparisons between medial and lateral type A photoreceptors were also significantly different $\left(0.4-0.6 \mathrm{spikes} / \mathrm{sec}, t_{15}=6.36\right.$, $p<0.005 ; 0.6-0.8$ spikes $/ \mathrm{sec}, t_{11}=3.15, p<0.01 ;>0.8$ spikes/ sec, $t_{20}=3.45, p<0.005$ ). The frequency of firing in the medial type A photoreceptor during stimulation of the medial B cell was significantly reduced over the three baseline frequency ranges studied. The frequency of firing in the lateral type A photoreceptor during stimulation of the lateral $B$ cell, however, was not significantly reduced in any of the three baseline frequency ranges studied. The difference in magnitude of current-elicited spike activity between the medial $A$ and the lateral $\Lambda$ photoreceptors indicates that the lateral A photoreceptor is affected less by the lateral B than the medial A photoreceptor is affected by the medial $\mathrm{B}$.
The mean percentage change in type A photoreceptor spike frequency in response to B cell stimulation is shown in Figure $8 B$. The lateral A continues to fire during lateral $B$ stimulation at or near the level of current elicited spike activity seen during baseline, while the medial A shows a significant decrease $(63 \%)$ in current-elicited spike activity during B stimulation $\left(t_{9}=4.85\right.$; $p<0.001$ ). This difference indicates that the medial B photoreceptor is significantly more effective in inhibiting spike activity in the medial A photoreceptor than the lateral B photoreceptors effect on the lateral A spike activity.

\section{Discussion}

The present study demonstrates that cellular correlates of classical conditioning are expressed differentially in identified type A photoreceptors. Of the two identified type A photoreceptors within each eye, only the lateral type A photoreceptor exhibited a statistically significant change in CS-elicited spike activity that was specific to CS-US pairings. In addition, we report for the first time evidence for enhanced excitability produced by conditioning in an identified type A photoreceptor. These results indicate that conditioning results in modifications of cellular mechanisms contributing to spike generation and alterations in spike frequency in addition to previously reported changes in membrane conductances that contribute to light-elicited generator potentials in isolated photoreceptors. Taken collectively, these findings have important implications for both cellular mcchanisms of conditioning in the CS pathway and potential alterations in the strength of synaptic connections between identified type A photoreceptors and postsynaptic neurons in the neural circuit supporting diminished phototactic behavior following conditioning.

\section{Correlates of conditioning in identified type A photoreceptors}

Our results show that statistically significant CS (light)-elicited increases in spike frequency are only found in lateral type A photoreceptors of conditioned animals. In contrast to the significant increase in CS-elicited activity of lateral type A photoreceptors, medial type A photoreceptors do not show significant changes in light-elicited activity in conditioned animals as compared to the pseudorandom controls. These findings indicate that cellular correlates of conditioning are differentially expressed in the two identified type A photoreceptors. Differences between our results and earlicr studics may bc due to experimental condition, for example, vertical versus horizontal orientation of the isolated nervous system, and axotomized versus synaptically intact photoreceptors. It was previously reported that light-elicited activity of type A photoreceptors was reduced in conditioned animals at all light intensities that were tested (Farley and Alkon, 1982). However, comparisons of spike frequency between identified medial and lateral type A photoreceptors were not conducted in the earlier study (Farley and Alkon, 1982). In addition, intracellular recordings were collected from photoreceptors in isolated nervous systems that were oriented vertically. Thus, the results of the Farley and Alkon study may be due to the contributions of increased inhibitory synaptic input from statocyst hair cells that would reduce the frequency of light-elicited action potentials recorded from type A photoreceptors.

In addition to CS-elicited enhanced spike activity of lateral type A photoreceptors, we found that the amplitude of the peak and plateau phase of generator potentials was significantly reduced in both medial and lateral type A photoreceptors of con- 


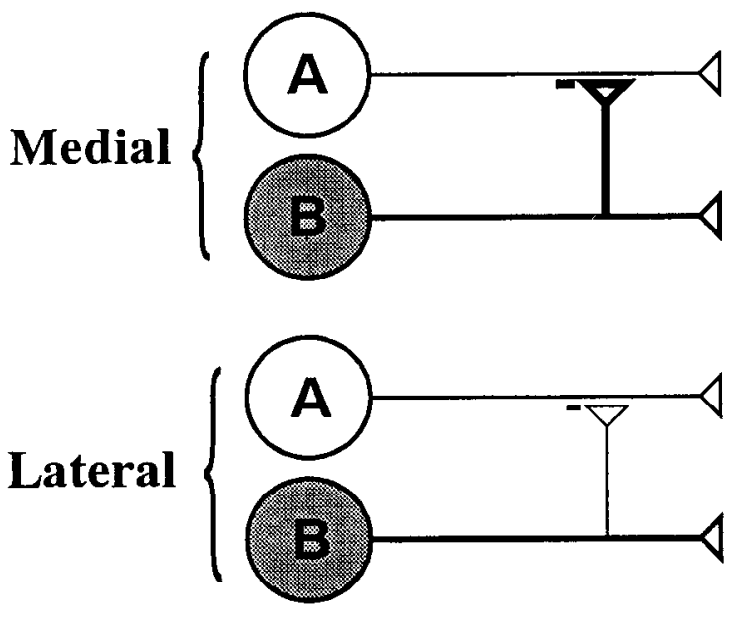

Figure 9. Circuit diagram illustrating the proposed differences in connections between medial and lateral type $A$ and $B$ photoreceptors. The inhibitory connection from the lateral B to the lateral A is weak. Stimulation of the lateral $B$ photoreceptor does not produce significant changes in the spike frequency of the lateral A photoreceptor. In contrast, the stronger inhibitory connection from the medial $B$ to the medial A results in significant inhibition of the spike frequency in the medial A photoreceptor.

ditioned animals. This aspect of our findings is consistent with a previous report showing that conditioning produced a reduction in the "steady-state" amplitude of generator potentials recorded from axotomized type A photoreceptors (Farley et al., 1990). Our present findings revealed significant differences between the paired and pseudorandom groups in the amplitude of the plateau phase of generator potentials measured at $30 \mathrm{sec}$, $60 \mathrm{sec}, 120 \mathrm{sec}$, and $5 \mathrm{~min}$ after light onset for medial type A photoreceptors. Lateral type A photoreceptors expressed significant differences between the paired and pseudorandom groups in the plateau phase only at $30 \mathrm{sec}$ and $60 \mathrm{sec}$ after light onset. Thus, medial type A photoreceptors are undergoing additional adaptation after $60 \mathrm{sec}$ of illumination. These results suggest that membrane conductances that are involved in the plateau phase of light-elicited generator potentials may contribute to these intrinsic changes produced by conditioning. A number of diverse $\mathrm{K}^{+}$conductances have been characterized in Hermissenda photoreceptors that may contribute to the plateau phase of the generator potential. Farley et al. (1990) have reported that the $\mathrm{Ca}^{2+}$-activated $\mathrm{K}^{+}$current $\left(I_{\mathrm{K}(\mathrm{Ca})}\right)$ is enhanced by conditioning, which would result in a reduction of the plateau phase of generator potentials. However, not all of the voltage-dependent $\mathrm{K}^{+}$conductances detected in the type A photoreceptors have been considered in previous analyses, especially the possible contribution of the delayed rectifier $I_{K(v)}$, which contributes to the plateau phase of the generator potential of type $B$ photoreceptors (Acosta-Urquidi and Crow, 1990). While the previous work and present results are consistent with the hypothesis that conditioning produces intrinsic changes (decreases) in the amplitude of the generator potential of type A photoreceptors, other mechanisms may be responsible for the enhanced excitability of lateral type A photoreceptors reported in this study, since an increase in an outward $\mathrm{K}^{+}$current would not be expected to enhance excitability or reduce spike frequency accommodation. Here we show that extrinsic current produces a significant increase in spike frequency of lateral type A photoreceptors of conditioned animals as compared to pseudorandom controls. The enhanced excitability was expressed over a broad range of depolarized membrane potentials. In addition, a significant decrease in accommodation was observed only in the lateral type A photoreceptors of conditioned animals. Clearly, different mechanisms must be responsible for the enhanced excitability of lateral type A photoreceptors and the reduction in the amplitude of the plateau phase of generator potentials. The reduction in the amplitude of the plateau phase of the generator potential of type A photoreceptors of conditioned animals reported by Farley et al. (1990) was observed in surgically axotomized preparations that do not support spike generation. Thus, the mechanism responsible for the reduction in the generator potential can be expressed in the absence of spike generation and synaptic input. In the present study, the photoreceptors were intact, where synaptic interactions and spike generation are normal. However, it is unlikely that enhanced synaptic inhibition of lateral type A photoreceptors could account for the enhanced excitability observed in our study, since the inhibitory synaptic connections between the lateral B and lateral A photoreceptor are weak (see below). Our present findings have implications for models of conditioning in Hermissenda that have attempted to explain the circuit interactions supporting conditioned modification of phototaxis. It was previously assumed that medial type A photoreceptors expressed a decrease in excitability in conditioned animals since the generator potential was reduced (Alkon, 1984). Our results show enhanced excitability that is expressed only in the lateral type A photoreceptor, and this identified photoreceptor exhibits correlates of conditioning that are specific to CS-IIS pairings. Interestingly, a dissociation between modifications of the generator potential amplitude and changes in light-elicited spike frequency were observed in type B photoreceptors of conditioned animals (Crow, 1985).

\section{Synaptic interactions within the visual system}

Previous rescarch has identificd scveral correlates of conditioning in identified type B photoreceptors (Crow and Alkon, 1980; Farley and Alkon, 1982; West et al., 1982; Crow, 1985). Based in part on these earlier observations, it was hypothesized that the enhanced excitability of the medial type B photoreceptor produced greater synaptic inhibition of the medial type A photoreceptor of conditioned animals (Alkon, 1984). In addition, it was hypothesized that the decreased excitability of the medial A produced by greater synaptic inhibition from the medial B, in addition to the contribution of intrinsic reductions in the amplitude of the medial $\mathrm{A}$ generator potential following conditioning, would produce less synaptic excitation of motor neurons (Goh and Alkon, 1984; Goh et al., 1985). Indirect projections from medial type $A$ photoreceptors to a putative motor neuron have been reported (Goh and Alkon, 1984). However, significant reductions in the spike frequency of identified medial type A photoreceptors elicited by the CS in conditioned animals have not been reported. Based upon the present findings, the earlier hypothesis must be modified, since CS-elicited spike frequency in the medial $\mathrm{A}$ is not significantly different in conditioned animals as compared to pseudorandom controls, and thus enhanced synaptic inhibition of putative motor neurons would not occur. Our findings indicate that the lateral $A$ is the only type A photoreceptor that encodes for the pairing of the CS and US. Thus, if type A photoreceptors contribute to phototactic suppression, the interneurons that receive synaptic input from the lateral type A photoreceptor would have information 
concerning pairing specificity. However, neural correlates of conditioning detected in putative motor neurons are nol consistent with the hypothesis that type A photoreceptor activity is the only critical event in phototactic suppression (Hodgson and Crow, 1992). Recent reports have suggested that CS-elicited activity recorded from pedal neurons or multiunit activity recorded from pedal nerves is reduced below the frequency of spontaneous activity recorded in the dark (Crow, 1981; Richards and Farley, 1987; Hodgson and (row, 1991, 1992). Because type A photoreceptors do not discharge action potentials spontaneously in the dark, their activity in response to light cannot be less than their activity in the dark. Taken collectively, the evidence suggests that both identified type B and type A photoreceptors may contribute to alterations in the activity of target neurons contributing to the circuitry controlling visually guided locomotion.

\section{Strength of synaptic interactions between identified pairs of type $A$ and $B$ photoreceptors}

CS-elicited activity of identified medial and lateral type A photoreceptors from synaptically intact preparations may be affected by synaptic interactions between photoreceptors within the eye. Previous work has shown that photoreceptors are mutually inhibitory, although it is not clear if the strength of the inhibition is invariant for each identified type of photoreceptor (Alkon and Fuortes, 1972). Our results show that extrinsic current elicited more action potentials in latcral type A photoreceptors of conditioned animals. The response to extrinsic current most likely involves membrane conductances that may contribute to the frequency of action potential generation rather than activating mutually inhibitory synaptic influences. In addition, differences in synaptic strength between identified pairs of type $\mathrm{A}$ and $\mathrm{B}$ photoreceptors exist prior to conditioning (see Fig. 9). Lateral B photoreceptors are weakly connected to lateral A photoreceptors, and thus enhanced activity of the lateral B photoreceptor would be expected to produce only minor inhibition of the lateral A photoreceptor. Indeed, our results show that the lateral $\mathrm{B}$ was only marginally effective in reducing the activity of the lateral A photoreceptor (see Results). In contrast, medial B photoreceptors produce strong synaptic inhibition of medial A photoreceptors, and thus conditioning correlates consisting of an enhancement of the generator potential of type B photoreceptors should enhance the medial B photoreceptors inhibition of the medial A photoreceptor. Such an outcome would be expected to counteract any enhanced excitability intrinsic to the medial A produced by conditioning. In fact, recent evidence suggests that IPSP amplitude may be enhanced by conditioning only in the medial B to medial A connection (Frysztak and Crow, 1992). In contrast, alterations in synaptic strength between lateral B and lateral A photoreceptors would have marginal effects because of the weak connections that are typically found between these identified pairs of photoreceptors.

\section{References}

Acosta-Urquidi J, Crow T (1990) 5-HT modulates two distinct $\mathrm{K}^{+}$ currents in Hermissenda type B photoreceptors. Soc Neurosci Abstr $16: 20$.
Alkon DL (1984) Calcium-mediated reduction of ionic currents: a biophysical memory trace. Science 225:1037-1045.

Alkon DL, Fuortes MGF (1972) Responses of photoreceptors in Hermissenda. J Gen Physiol 60:631-649.

Alkon DL, Lederhendler I, Shoukimas JJ (1982) Primary changes of membrane currents during retention of associative learning. Science 215:693-695.

Alkon DL, Sakakibara M, Forman R, Harrigan J, Lederhendler I, Farley J (1985) Reduction of two voltage-dependent $\mathrm{K}^{+}$currents mediates retention of a learned association. Behav Neural Biol 44:278-300.

Crow T (1981) Neurophysiological correlates of conditioning in identified putative motor neurons in Hermissenda. Soc Neurosci Abstr $7: 352$.

Crow T (1983) Conditioned modification of locomotion in Hermissenda crassicornis: analysis of time-dependent associative and nonassociative components. J Neurosci 3:2621-2628.

Crow T (1985) Conditioned modification of phototactic behavior in Hermissenda. II. Differential adaptation of B photoreceptors. J Neurosci 5:215-223.

Crow T (1988) Cellular and molecular analysis of associative learning and memory in Hermissenda. Trends Neurosci 11:136-142.

Crow T, Alkon DL (1978) Retention of an associative behavioral change in Hermissenda. Science 201:1239-1241.

Crow T, Alkon DL (1980) Associative behavioral modification in Hermissenda: cellular correlates. Science 209:412-414.

Crow T, Forrester J (1991) Light paired with serotonin in vivo produces both short- and long-term enhancement of generator potentials of identified B photoreceptors in Hermissenda. J Neurosci 11:608-617.

Crow T, Offenbach N (1983) Modification of the initiation of locomotion in Hermissenda: behavioral analysis. Brain Res 271:301-310.

Farley J, Alkon DL (1982) Associative neural and behavioral change in Hermissenda: consequences of nervous system orientation for lightand pairing-specificity. J Neurophysiol 48:785-807.

Farley J, Alkon DL (1985) Cellular mechanisms of learning, memory, and information storage. Annu Rev Psychol 36:419-464.

Farley J, Richards WG, Grover LM (1990) Associative learning changes intrinsic to Hermissenda type A photoreceptors. Behav Neurosci 104: 135-152.

Frysztak RJ, Crow T (1991) Correlates of conditioning in pairs of identified type A and B photoreceptors in Hermissenda: effects of adaptation, light intensity and synaptic interactions. Soc Neurosci Abstr 17:15.

Frysztak RJ, Crow T (1992) Synaptic interactions between pairs of identified type A and $\mathrm{B}$ photorcceptors in conditioncd Hermissenda. Soc Neurosci Abstr 18:15.

Goh Y, Alkon DL (1984) Sensory, interneuronal, and motor interactions within Hermissenda visual pathway. J Neurophysiol 52:156168.

Goh Y, Lederhendler I, Alkon DL (1985) Input and output changes of an identified neural pathway are correlated with associative learning in Hermissenda. J Neurosci 5:536-453.

Hodgson TM, Crow T (1991) Characterization of 4 light-responsive putative motor neurons in the pedal ganglia of Hermissenda crassicornis. Brain Res 557:255-264.

Hodgson TM, Crow T (1992) Cellular correlates of classical conditioning in identified light responsive pedal neurons of Hermissenda crassicornis. Brain Res 570:267-271.

Lederhendler II, Gart S, Alkon DL (1986) Classical conditioning of Hermissenda: origin of a new response. J Neurosci 6:1325-1331.

Richards WG, Farley J (1987) Motor correlates of phototaxis and associative learning in Hermissenda. Brain Res Bull 19:175-189.

West A, Barnes ES, Alkon DL (1982) Primary changes of voltage responses during retention of associative learning. J Neurophysiol 48: 1243-1255.

Winer BJ (1962) Statistical principles in experimental design. New York: McGraw-Hill. 